
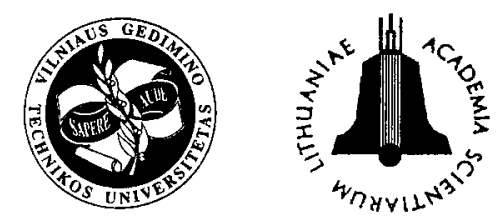

JOURNAL OF CIVIL ENGINEERING AND MANAGEMENT

http:/www.vtu.lt/english/edition

2002, Vol VIII, No 2, 104-107

\title{
SHAKEDOWN OF BOLTS WITH A ONE-SIDED PROPAGATING CRACK
}

\author{
Mindaugas Leonavičius', Marijonas Šukšta ${ }^{2}$ \\ Dept of Strength of Materials, Vilnius Gediminas Technical University, \\ Sauletekio al.11,LT-2040Vilnius, Lithuania. ${ }^{1}$ E-mail: minleo@fm.vtu.lt ${ }^{2}$ E-mail: marsu@fm.vtu.lt
}

Received 28 June 2001; accepted 17 Jan 2002

\begin{abstract}
Cyclic strains of joints may cause formation of a crack or cracks in a bolt thus changing its loading conditions. After crack appearance in a bolt it can be used if its length does not exceed a critical one. Modification of the shakedown theorem - the method of additional load - was applied in the investigation. The results - the analytical expressions of shakedown conditions are in a sufficiently good agreement with those achieved by other methods. Differences occur due to failure to get an accurate analytic expression. Solution of the shakedown problem plays an essential role in determining the safety factor of bolts subjected to cyclic strains.
\end{abstract}

Keywords: fatigue, crack, shakedown, bolts.

\section{Introduction}

Conditions that are sufficient for the development of shakedown are validated by the Melan's principle (the static theorem of shakedown) and Coiter's kinematic theorem. The further investigations of shakedown theories are presented in works of G. Maier, J. König, G. Polizzoto, A. Čižas, J. Atkočiūnas, etc.

If at the beginning of loading residual deformations due to plastic yield stop accumulating after a certain number of cycles, it means that a construction has reached its shakedown state under a given load. An analysis of shakedown conditions may help determine the limit values of external parameters [1-5].

An ideal material without any defect does not exist in reality. Provided defect (crack) is propagating, the rupture risk and evolution of shakedown conditions have to be investigated. An new extensions of shakedown theory for cracked bodies are proposed in [6-8].

The analytical expressions [9-12] derived and shakedown diagrams developed on their basis are used to elaborate a reserve calculation procedure for a progressive profile change in different threaded joints (without a crack, with one- and double-sided cracks). Evaluation of the threaded joint shakedown process and determination of the safety factor are matters of great importance.

The shakedown of a bolt with a double-sided crack has been previously investigated [10,12]. Modification of the static theorem of shakedown - the method of additional load - was applied in the investigation [10]. In the present work this method is used for a bolt subjected to a complex deformation when a one-sided fatigue crack propagates over the cross-section of the bolt.

\section{The investigation aim}

The aim of the work was to define the shakedown area of a bar with a circular cross-section (a bolt or a stud) in case of formation and propagation of a crack on one side of a bolt. The bolt was deformed by a constant axial force within symmetrically constant limits at a variable bending moment, ie, $N=$ const, while $-M^{*} \leq M \leq M^{*}, M^{*}=$ const. The bolt material was perfectly elastoplastic.

\section{Geometrical properties of the cross-section}

The method of additional load is quite frequently used, and the results of this method are verified by solving problems using other methods. Application of this method does not cause too great difficulties; however, when it is applied to a bar with a one-sided crack, the problem of an analytical solution arises.

The cross-section of a bar with a crack of the depth $h_{p}$ is shown in Fig 1. The crack depth is also defined by the angle $\alpha_{p}$. Some intermediate operations were left out, and the main geometrical indices necessary for the analysis were deduced.

The area of the crack-free section of the circle is:

$$
\begin{aligned}
A_{S} & =\int_{A} d A=\int_{-r}^{r-h_{p}} b \cdot d x=2 \cdot r^{2} \int_{\alpha_{p}}^{\pi} \cdot \sin ^{2} \alpha \cdot d \alpha= \\
& =r^{2} \cdot\left(\pi-\alpha_{p}+\sin \alpha_{p} \cdot \cos \alpha_{p}\right) .
\end{aligned}
$$

The area of the crack zone is:

$$
A_{p}=r^{2} \cdot\left(\alpha_{p}-\sin \alpha_{p} \cdot \cos \alpha_{p}\right) \text {. }
$$




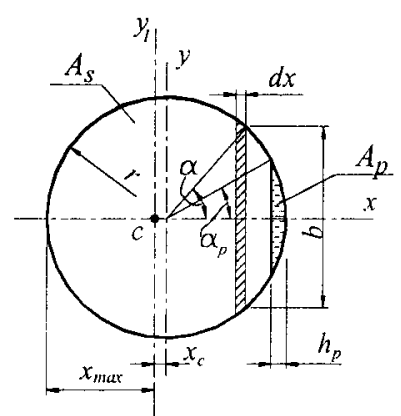

Fig 1. Bolt cross-section with a crack

The co-ordinate of the cross-section centre of a crack weakened bar is:

$$
x_{c}=-\frac{S_{y}}{A_{s}}=-\frac{2}{3} \cdot \frac{\sin ^{3} \alpha_{p}}{\pi-\alpha_{p}+\sin \alpha_{p} \cdot \cos \alpha_{p}} \cdot r .
$$

The second moment of area in respect of the central axis $y_{1}$ is:

$$
\begin{aligned}
& I_{y 1}=I_{y}-x_{c}^{2} \cdot A_{s}= \\
& =\frac{\pi r^{4}}{4} \cdot\left(1-\alpha_{p}+2 \sin \alpha_{p} \cos ^{3} \alpha_{p}-\right. \\
& \left.\sin \alpha_{p} \cos \alpha_{p}\right)-\frac{4}{9} \cdot r^{4} \cdot \frac{\sin ^{6} \alpha_{p}}{\pi+\sin \alpha_{p} \cos \alpha_{p}-\alpha_{p}} .
\end{aligned}
$$

Section modulus is:

$$
\begin{aligned}
& W_{y 1}=r^{3} \cdot\left(\frac{1}{4} \pi+\frac{1}{2} \sin \alpha_{p} \cos ^{3} \alpha_{p}-\right. \\
& \frac{1}{4} \sin \alpha_{p} \cos \alpha_{p}-\frac{1}{4} \alpha_{p}- \\
& \left.\frac{4}{9} \cdot \frac{\sin ^{6} \alpha_{p}}{\pi+\sin \alpha_{p} \cos \alpha_{p}-\alpha_{p}}\right) /(1- \\
& \left.\frac{2}{3} \cdot \frac{\sin ^{3} \alpha_{p}}{\pi+\sin \alpha_{p} \cos \alpha_{p}-\alpha_{p}}\right) .
\end{aligned}
$$

In addition to these geometrical indicators, the "plastic" section modulus of the cross-section $W_{p l}$ is necessary, which is equal to the sum of the static moments of the stretched and compressed zone in respect of the neutral axis. In bending case and when a perfectly elastoplastic material yields in the whole area of the crosssection, the neutral axis divides the cross-section into two equal areas. Application of this generally known law causes certain difficulties in attempts to define shakedown conditions by means of analytic equations. In solving this problem failure to obtain an analytical solution of the neutral axis position is unavoidable. In this case the dependence of the neutral axis upon the crack angle $\alpha_{p}$ was expressed approximately by a second-order polynomial function. In spite of the simplicity of the function of the neutral axis position, the obtained analytic expression of the "plastic" section modulus is very complicated, therefore it is not presented in this paper.

\section{Conditions of shakedown}

The geometrical properties described above are sufficient for a bolt shakedown analysis. Appearance of alternating plastic deformation would be the first fracture case of a deformed bar. Alternating plastic deformation under the action of a variable bending moment will occur, if the bending moment reaches the limiting value $M_{e}$ which is further conditionally referred to as the limit elastic moment. It is equal to:

$$
M_{e}=W_{y l} \cdot \sigma_{y}
$$

where $\sigma_{y}$ is the yield stress.

Thus the condition of alternating plastic deformation would be:

$$
M=M_{e} \text { or } \frac{M}{M_{e}}=1 .
$$

It is convenient to express the limit elastic moment $M_{e}$ by the limit moment of the non-weakened crosssection $M_{y}^{0}$, which causes yield in the whole cross-section and is equal to:

$$
M_{y}^{0}=\frac{4}{3} r^{3} \sigma_{y} .
$$

After defining the ratio $s_{m}$ between the limit moments $M_{e}$ and $M_{y}^{0}$ from the expressions (6) and (8), we receive the following:

$$
M_{e}=s_{m} M_{y}^{0}
$$

where

$$
\begin{aligned}
& s_{m}=\frac{3}{4}\left(\frac{\pi}{4}+\frac{\sin \alpha_{p} \cos ^{3} \alpha_{p}}{2}-\right. \\
& \frac{\sin \alpha_{p} \cos \alpha_{p}}{4}-\frac{\alpha_{p}}{4}- \\
& \left.\frac{4}{9} \cdot \frac{\sin ^{6} \alpha_{p}}{\pi+\sin \alpha_{p} \cos \alpha_{p}-\alpha_{p}}\right) /\left(1-\frac{2}{3} .\right. \\
& \left.\frac{\sin ^{3} \alpha_{p}}{\pi+\sin \alpha_{p} \cos \alpha_{p}-\alpha_{p}}\right) .
\end{aligned}
$$

After putting the expression (9) into the formula (7), we obtain the following condition of alternating plastic deformation:

$$
\frac{M}{s_{m} M_{y}^{0}}=1
$$

The ratio $M / M_{y}^{0}$ is the relative co-ordinate of the bending moment. After marking it as $m$, the dependence (11) could be put down as follows: 
Table 1. Dependence of the relative crack depth $k$ on the coordinate $m$ :

\begin{tabular}{|c|c|c|c|c|c|c|c|c|c|c|}
\hline$k$ & 0 & 0.05 & 0.10 & 0.15 & 0.20 & 0.25 & 0.30 & 0.35 & 0.40 & 0.45 \\
\hline$m$ & 0.589 & 0.578 & 0.559 & 0.537 & 0.513 & 0.488 & 0.462 & 0.436 & 0.409 & 0.383 \\
\hline
\end{tabular}

$$
m=s_{m}
$$

In the Fig 2 straight line 1 depicts the alternating yield condition. After expressing the crack depth by a relative value $k=h_{p} / r$, the coordinate $m$ is calculated and the zone, in which the bar would adapt itself to alternating plastic deformation, is determined. This calculation is presented in Table 1.

The second plastic fracture mechanism will appear when the plastic deformation zone of the cross-section crosses the previous neutral axis at each half-cycle of the moment change. Then plastic deformation will start ac-cumulating at the cross-section centre, and plastic fracture will develop. Using the principles of the additional load method, we may add to the axial force $N$ such an of axial force increase $\Delta N$ which would cause total plastic deformation of the bar only due to axial force:

$$
N+\Delta N=N_{y},
$$

where $N_{y}=A_{s} \sigma_{y}$ is the limit axial force causing plastic deformation of the whole cross-section; $A_{s}$ is the cross-section area calculated by means of the formula (1).

The axial force increase $\Delta N$ without evaluation of unloading occurring on one side of the cross-section, may be expressed by the bending moment increase $\Delta M$ :

$$
\Delta N=\int_{A_{1}} \sigma d A=\int_{A_{1}} \frac{\Delta M}{I_{y 1}} x d A=\frac{\Delta M}{I_{y 1}} \int_{A_{1}} x d A=\frac{\Delta M}{I_{y 1}} \cdot S_{n l}^{*}
$$

where $S_{n l}^{*}$ is the first moment of half of the cross-section in respect of the neutral axis.

The increase of bending moment is equal to a double limit of its change ie $\Delta M=2 M^{*}$, and the expression of the second moment of area is presented by the formula (4). By putting these values into the formula (14) and integrating it we obtain the following:

$$
\Delta N=M^{*} \gamma,
$$

where $\gamma$ is a respective factor obtained by integrating the expression (14).

Now the expression (15) is put into the formula (13):

$$
N+M^{*} \gamma=N_{y} \quad \text { or } \quad \frac{N}{N_{y}}+\frac{M^{*}}{N_{y}} \gamma=1
$$

Here the term $N_{y}$ may be expressed by the limit moment $M_{y}$ causing the total yield of the cross-section damaged by a crack:

$$
M_{y}=W_{p l} \sigma_{y}
$$

After marking $N_{y} / M_{y}$ as b and putting the expression of $N_{y}$ into the formula (16), we obtain:

$$
\frac{N}{N_{y}}+\frac{M}{M_{y}} \cdot \frac{\gamma}{\beta}=1
$$

where $M$ is the bending moment which in the limit case is equal to the limiting value $M^{*}$ of the bending moment. The limit stresses $N_{y}$ and $M_{y}$ of the weakened crosssection may be replaced by the limiting stresses of the whole cross-section $N_{y}^{0}=\pi r^{2} \sigma_{y}$ and $M_{y}^{0}$. The latter value is calculated by means of the formula (8). After marking the respective factors as $k_{n}$ and $k_{m}$, the stresses of the weakened cross-section are expressed in the following way:

$$
\begin{aligned}
& N_{y}=k_{n} N_{y}^{0}, \\
& M_{y}=k_{m} M_{y}^{0} .
\end{aligned}
$$

By putting the expression (19) into the formula (18) we obtain:

$$
\frac{N}{N_{y}^{0}} \cdot \frac{1}{k_{n}}+\frac{M}{M_{y}^{0}} \cdot \frac{\gamma}{k_{m} \beta}=1
$$

Regarding that $N_{y} / N_{y}^{0}$ and $M_{y} / M_{y}^{0}$ are the relative stress coordinates $n$ and $m$, the formula (20) will finally obtain the following expression:

$$
n \frac{1}{k_{n}}+m \frac{\gamma}{k_{m} \beta}=1 \text { or } a \cdot n+b \cdot m=1 \text {. }
$$

The latter equation describes the bar shakedown condition in case of the second plastic fracture. These equations are presented in Table 2 according to the relative crack depth $k$.

Table 2. Equations of the relative crack depth $\boldsymbol{k}$ and conditions of progressing plastic fracture

\begin{tabular}{|c|c|}
\hline $\begin{array}{c}\text { Relative crack } \\
\text { depth } k\end{array}$ & Conditions of progressing plastic fracture \\
\hline 0 & $n+0.725 m=1$ \\
\hline 0.05 & $1.007 n+0.733 m=1$ \\
\hline 0.10 & $1.019 n+0.734 m=1$ \\
\hline 0.15 & $1.035 n+0.782 m=1$ \\
\hline 0.20 & $1.055 n+0.816 m=1$ \\
\hline 0.25 & $1.077 n+0.854 m=1$ \\
\hline 0.30 & $1.104 n+0.898 m=1$ \\
\hline 0.35 & $1.133 n+0.948 m=1$ \\
\hline 0.40 & $1.166 n+1.004 m=1$ \\
\hline 0.45 & $1.202 n+1.067 m=1$ \\
\hline 0.50 & $1.243 n+1.138 m=1$ \\
\hline
\end{tabular}




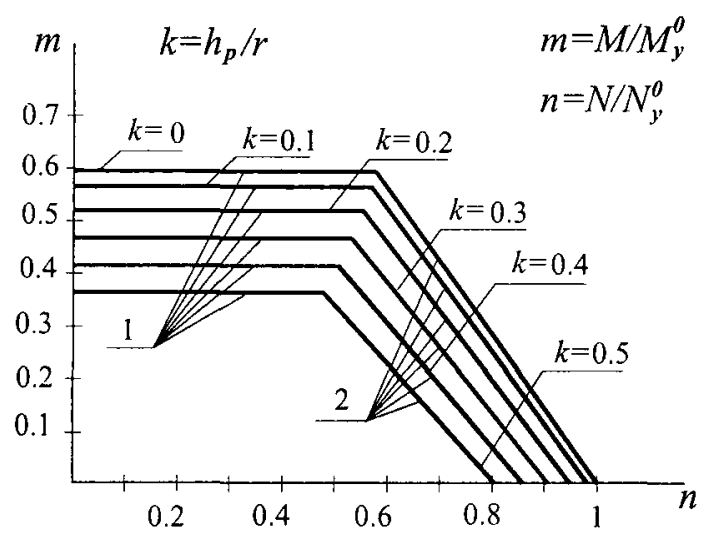

Fig 2. Dependence of the cracked bolt shakedown zone on the crack depth

Depending on the crack depth, the bolt shakedown zone may be drawn in the area of stresses (Fig 2). Straight lines 2 define the zone that limits progressing plastic fracture, and the zone of alternating plastic deformation is described by straight lines 1 .

\section{Conclusions}

Application of the additional load method for defining the shakedown of a bolt with a propagating crack is quite simple and effective. The obtained results are the analytical expressions of shakedown conditions for a bar with one-sided crack. Those findings are in a sufficiently good agreement with those achieved by using other methods. Divergences are caused by failure to obtain an accurate analytical expression. On the other hand, even approximate analytical expressions obtained by using various programmes computational mathematics are very complicated. Although analytical expressions are of a generalising character, they loose, unfortunately, their expressivity because of their complexity. That is why the solution of this problem should be more accurate by using numerical methods. Solution of the shakedown problem is the first step in determining the safety factor of cyclically deformed bolts.

\section{References}

1. Gokhfeld D. A. Cherniavsky O. F. Limit analysis of structures at thermal cycling. Sijthoff and Noordhoff, Alphen aan den Rijn - The Netherlands, Rockville, Maryland USA, 1980. $537 \mathrm{p}$.

2. Atkočiūnas J. New approach to the Koiter's inequality for shakedown. Mechanics Research Communications, Vol 20, No 4, 1993, p. 301-308.

3. Atkočiūnas J. Design of elastoplastic systems under repeated load (Расчет упругопластических систем при повторном нагружении). Vilnius: Mokslas, 1994. 150 p. (in Russian).

4. Atkočiūnas J. Compatibility equations of strains for degenerate shakedown problems. Computer and Structures, Vol 63, No 2, 1997, p. 277-282.

5. Huang Y. and Stein E. An analytical method for shakedown problems with linear kinematic hardening material. International Journal of Solids and Structures, 31, 18 2433-2444, 1994.

6. Nguen Q. S. Methodes energetiques en mecanique de la rupture. Journal de Mecanique, Vol 19, No 2, 1980.

7. Huang Y. and Stein E. Shakedown of a cracked body consisting of kinematic hardening material. Engineering Fracture Mechanics, 54, 1, 107-112, 1996.

8. Belouchrani M. A. and Weichert D. An extension of the static shakedown theorem to inelastic cracked structures. International Journal of Mechanical Sciences, 41, 163$177,1999$.

9. Leonavičius M. Influence of Cyclic Bending on the Prestress and Shakedown of Threaded Joints. Mechanics (Mechanika), Nr. 1(12). Kaunas: Technologija, 1998, p. $11-$ 19.

10. Leonavičius M., Šukšta M. The bolt-with- a crack shakedown estimation by the method of additional load. Civil Engineering (Statyba), Nr. 1(9). Vilnius: Technika, 1997, p. $74-77$.

11. Leonavičius M. and Krenevičius A. Shakedown and Failure of the Threaded Joints under Low Cyclic Loading. Journal of Construction Steel Research, Vol 46. 1998. Steel Construction Institute. Elsevier, p. 452-453. Special issue on CDROM.

12. Leonavičius M., Šukšta M. The propagation of crack and the shakedown of threaded joints under low cyclic load. Mechanics (Mechanika), Nr. 3. Kaunas: Technologija, 1995, p. 16-18 (in Lithuanian). 